\title{
Minimizing Transfer Time to an ST Segment Elevation Myocardial Infarction Receiving Center: A Modified Delphi Consensus
}

\author{
Bryn E. Mumma, MD, MAS ${ }^{a}$, Conrad Williamson, BS ${ }^{b}$, Rahul K. Khare, MD, MS ${ }^{c}$, Kevin E. \\ Mackey, MD $^{\mathrm{d}}$, and Deborah B. Diercks, MD, MSc ${ }^{\mathrm{a}}$ \\ aDepartment of Emergency Medicine, University of California Davis, Sacramento, CA \\ bUniversity of California Davis School of Medicine, Sacramento, CA \\ 'Department of Emergency Medicine, Northwestern University, Chicago, IL \\ dDepartment of Emergency Medicine, Kaiser Permanente South Sacramento, Sacramento, CA
}

\begin{abstract}
Of patients with ST segment elevation myocardial infarction (STEMI), approximately two-thirds present to a hospital not capable of percutaneous coronary intervention. Transfer to a STEMI receiving center delays time to reperfusion in patients with STEMI, but factors that affect this delay have not been well studied. We performed a three-round modified Delphi study to identify system practices that minimize transfer time to a STEMI receiving center. A comprehensive literature review was used to identify candidate system practices. Emergency medical services, emergency medicine, and cardiology experts were invited to participate. Consensus was defined as $80 \%$ agreement that a variable was "very important (5)" or "important (4)" with a mean score $\geq 4.25$ or $80 \%$ agreement that a variable was "not important (1)" or "somewhat important (2)" with a mean score 4.75 . In Round 1, participants rated the candidate items and suggested additional items. Individual feedback was provided, and participants discussed items via conference calls before rating them again in Round 2. In Round 3, participants ranked the consensus items from Rounds 1-2 from most to least important, and the mean score for each item was calculated. Of the 98 experts invited, 29 participated in Round 1, 22 in Round 2, and 14 in Round 3. Participants identified 18 system practices that they agree are critical in minimizing transfer time to STEMI receiving centers, with the most important being performance of a prehospital electrocardiogram and having established transfer protocols. These factors should be considered in the development of STEMI systems of care.
\end{abstract}

\section{Keywords}

STEMI; regionalization; acute myocardial infarction; door-to-balloon time

Address for Correspondence: Bryn E. Mumma, UC Davis Department of Emergency Medicine, 4150 V Street, PSSB \#2100, Sacramento, CA 95817, Phone (916) 734-5010, Fax (916) 734-7950, mummabe@ gmail.com. 


\section{Introduction}

Approximately 250,000 patients suffer from an ST segment elevation myocardial infarction (STEMI) each year in the United States. ${ }^{1}$ Of these patients, over three-quarters present to the hospital via private vehicle rather than via emergency medical services (EMS), ${ }^{2}$ and approximately two-thirds receive initial care at a hospital without primary percutaneous coronary intervention (PCI) capability. ${ }^{3}$ Timely PCI for patients with STEMI reduces mortality and morbidity. ${ }^{4}$ American College of Cardiology (ACC)/American Heart Association (AHA) guidelines recommend total ischemic time of less than 120 minutes from initial EMS contact and less than 90 minutes from hospital door to balloon. ${ }^{5}$ Additionally, the AHA's Mission:Lifeline program recommends a door-in-door-out time at the STEMI referral hospital of less than 45 minutes. ${ }^{6}$ Transfer from a STEMI referral hospital to a STEMI receiving center has been shown to delay time to reperfusion in patients with STEMI, with as few as $6.5 \%$ of transferred patients meeting the 90 -minute door to balloon goal. $^{7}$

STEMI receiving center strategies for reducing time to reperfusion have been studied, but few investigations have focused on the entire STEMI system of care, which includes EMS and STEMI referral hospitals. ${ }^{8}$ Furthermore, the AHA recognizes that "although some regions have successfully adopted a STEMI systems approach, there currently is no data repository in which to catalog examples of protocols used or transfer policies or to review assessment of why elements of the STEMI system succeeded or failed in a region." "9

The objective of this study is to identify system practices that minimize the transfer time to a STEMI receiving center for patients who present to a STEMI referral hospital.

\section{Methods}

\section{Study Design}

We utilized a modified Delphi technique to identify expert consensus on factors important in minimizing transfer time to STEMI receiving center for patients who present via emergency medical services (EMS) or private vehicle to a STEMI referral hospital. Transfer time was defined as the time from patient arrival at a STEMI referral hospital to arrival at a STEMI receiving center. This method uses a systematic approach to achieve consensus among a panel of experts on a topic where existing knowledge is incomplete..$^{10}$ This study was approved by the University of California Davis Institutional Review Board.

\section{Study Setting and Population}

Expert participants were identified by three mechanisms: (1) first or senior author on a study addressing barriers to timely transport of STEMI patients to a STEMI receiving center, (2) panelist on AHA Mission Lifeline regional committees, and/or (3) recommendation from an individual meeting one of the criteria above. Efforts were made to include individuals with expertise in EMS systems, emergency medicine, and interventional cardiology from diverse geographic and clinical settings. The study investigators served as process moderators and did not contribute their opinions to the process. Potential participants were emailed two invitations to participate. 


\section{Study Protocol}

Candidate prehospital, STEMI referral hospital, and STEMI receiving center processes that minimize transfer time were identified via literature review.

In Round 1, participants rated the importance of each of the candidate processes with regards to minimizing transfer time using a five-point Likert scale with the options "Very important" (5), "Important" (4), "Don't know/Neutral" (3), "Somewhat important" (2), "Not at all important" (1). Participants were invited to suggest additional items for consideration. Aggregate and individual response data were distributed to each participant, and participants discussed all items in conference calls moderated by the investigators. The goals of the conference calls were to allow participants to present the rationale for their ratings, to debate the importance of candidate items, and to refine the additional items suggested during Round 1 . To preserve anonymity, participants identified themselves by number during the conference calls.

In Round 2, participants rated each of the non-consensus items from Round 1 as well as the items suggested by panelists during Round 1 using the same five-point Likert scale.

In Round 3, participants ranked consensus items from Rounds 1 and 2 in order of their importance for minimizing transfer time. Final rank order was determined by each item's mean rank score.

\section{Measurements or key outcome measures}

The primary outcome was expert consensus on system processes. Consensus was defined as $80 \%$ agreement that an item was "Very important (5)" or "Important (4)" with a mean score $\geq 4.25$ or $80 \%$ agreement that an item was "Not at all important (1)" or "Somewhat important (2)" with a mean $\unlhd .75$. When participants reached consensus that an item was not important, the item was removed from further discussion. In Round 3, mean rank score was calculated by adding all respondents' scores for each item and dividing by the number of respondents.

\section{Data Analysis}

Summary and descriptive statistics were performed using Microsoft Excel 2010 (Microsoft, Redmond, WA).

\section{Results}

Ninety-eight experts were invited to contribute; twenty-nine participated in Round 1, twenty-two in Round 2, and fourteen in Round 3. Only those experts who participated in Round 1 were invited to contribute in Rounds 2-3. Overall, 23\% (15/65) of responses were from interventional cardiologists. (Table 1.)

Thirty candidate system processes reported to affect transfer time were identified during literature review. These included 8 prehospital processes and 22 hospital processes. Six patient factors were also evaluated. During Round 1, experts suggested four additional EMS processes, 7 additional hospital processes, and 5 additional patient factors. 
During Rounds 1 and 2, participants reached on consensus on 18 items felt to be important for minimizing transfer time and two items felt not to be important for minimizing transfer time. (Figure 1.) In Round 3, the most important system practices for minimizing transfer time from a STEMI referral hospital to a STEMI receiving center were prehospital providers performing an electrocardiogram and STEMI referral hospitals and receiving centers having an established transfer protocol. (Table 2.) Participants also reached consensus agreement that patient insurance status was not important in determining transfer time from a STEMI referral hospital to a STEMI receiving center.

\section{Limitations}

Our data are limited by our survey response rate; however, participants included experts from EMS systems, emergency medicine, and interventional cardiology. While our results may not be generalizable to all settings, we included participants from varied practice environments to generate processes that are applicable to a broad range of STEMI systems. Lastly, the impact of each of these strategies on actual transfer times from STEMI referral hospitals to STEMI receiving centers remains unknown.

\section{Discussion}

Of 41 systems processes evaluated by participants, consensus was achieved on 20 items, with 18 felt to be important and two deemed unimportant. The most important EMS processes focused on early recognition of STEMI and notification of the STEMI referral hospital. While participants agreed that performance of prehospital electrocardiograms (ECGs) was critical, they disagreed on the importance of paramedic versus machine interpretation of the ECG and generally felt that this distinction should be made by individual EMS systems based on available resources. These EMS actions enable the STEMI referral hospital to prepare for the patient's arrival and to initiate downstream steps in the protocol.

Consensus hospital processes centered on having established protocols with dedicated resources for the care of STEMI patients at the STEMI referral hospital. Participants also emphasized the importance of minimizing administrative tasks related to patient transfer, and they felt that dedication to the AHA goals and strong communication within the STEMI system with regards to individual patients and overall system performance were key components. These themes are similar to those identified in a meta-analysis of system processes to reduce door-to-balloon times at STEMI receiving centers. ${ }^{8}$

One quality improvement protocol designed to reduce time to reperfusion for patients with STEMI is North Carolina's statewide RACE program. During this program, patients transferred from a STEMI referral hospital to a STEMI receiving center had a median doorin-door-out time of 67 minutes and a median time from first medical contact to PCI of 152 minutes. ${ }^{7}$ These data suggest that additional strategies are needed to reach the AHA goals for transferred patients. Participants in our study agreed that seven of the eight system processes recommended in the RACE program ${ }^{11}$ were important, and they identified ten 
additional processes that may enable systems to meet AHA time goals for transferred STEMI patients.

By integrating expert opinions from EMS, emergency medicine, and interventional cardiology, we propose a set of system processes to minimize the transfer time from a STEMI referral hospital to a STEMI receiving center.

\section{Conclusions}

Expert participants identified 18 system practices that they agree are critical in minimizing transfer time to STEMI receiving centers. These factors should be considered in the development of STEMI systems of care.

\section{Acknowledgments}

Source of Funding: The first author is supported by the National Heart, Lung, and Blood (NHLBI) Research Career Development Programs in Emergency Medicine through grant \#5K12HL108964. The project described was supported by the National Center for Advancing Translational Sciences, National Institutes of Health, through grant \#UL1 TR000002. The content is solely the responsibility of the authors and does not necessarily represent the official views of the National Institutes of Health.

\section{References}

1. Roger VL, Go AS, Lloyd-Jones DM, et al. Heart disease and stroke statistics--2012 update: a report from the American Heart Association. Circulation. 2012; 125:e2-e220. [PubMed: 22179539]

2. Jacobs AK, Antman EM, Faxon DP, Gregory T, Solis P. Development of systems of care for STelevation myocardial infarction patients: executive summary. Circulation. 2007; 116:217-30. [PubMed: 17538045]

3. Henry TD, Atkins JM, Cunningham MS, et al. ST-segment elevation myocardial infarction: recommendations on triage of patients to heart attack centers: is it time for a national policy for the treatment of ST-segment elevation myocardial infarction? J Am Coll Cardiol. 2006; 47:1339-45. [PubMed: 16580518]

4. Berger PB, Ellis SG, Holmes DR Jr, et al. Relationship between delay in performing direct coronary angioplasty and early clinical outcome in patients with acute myocardial infarction: results from the global use of strategies to open occluded arteries in Acute Coronary Syndromes (GUSTO-IIb) trial. Circulation. 1999; 100:14-20. [PubMed: 10393675]

5. Kushner FG, Hand M, Smith SC Jr, et al. 2009 focused updates: ACC/AHA guidelines for the management of patients with ST-elevation myocardial infarction (updating the 2004 guideline and 2007 focused update) and ACC/AHA/SCAI guidelines on percutaneous coronary intervention (updating the 2005 guideline and 2007 focused update) a report of the American College of Cardiology Foundation/American Heart Association Task Force on Practice Guidelines. J Am Coll Cardiol. 2009; 54:2205-41. [PubMed: 19942100]

6. [July 1, 2013] Mission: Lifeline PARTICIPATION Requirements for STEMI Systems of Care. 2013. at http://www.heart.org/idc/groups/heart-public/@wcm/@hcm/@ml/documents/ downloadable/ucm_438840.pdf.

7. Fosbol EL, Granger CB, Peterson ED, et al. Prehospital system delay in ST-segment elevation myocardial infarction care: a novel linkage of emergency medicine services and in hospital registry data. Am Heart J. 2013; 165:363-70. [PubMed: 23453105]

8. McDermott KA, Helfrich CD, Sales AE, Rumsfeld JS, Ho PM, Fihn SD. A review of interventions and system changes to improve time to reperfusion for ST-segment elevation myocardial infarction. J Gen Intern Med. 2008; 23:1246-56. [PubMed: 18459014] 
9. Solis P, Amsterdam EA, Bufalino V, Drew BJ, Jacobs AK. Development of systems of care for STelevation myocardial infarction patients: policy recommendations. Circulation. 2007; 116:e73-6. [PubMed: 17538035]

10. Hsu CC, Sandford BA. The Delphi Technique: Making Sense of Consensus. Practical Assessment, Research \& Evaluation. 2007; 12:1-8.

11. Glickman SW, Lytle BL, Ou FS, et al. Care processes associated with quicker door-in-door-out times for patients with ST-elevation-myocardial infarction requiring transfer: results from a statewide regionalization program. Circ Cardiovasc Qual Outcomes. 2011; 4:382-8. [PubMed: 21712523] 


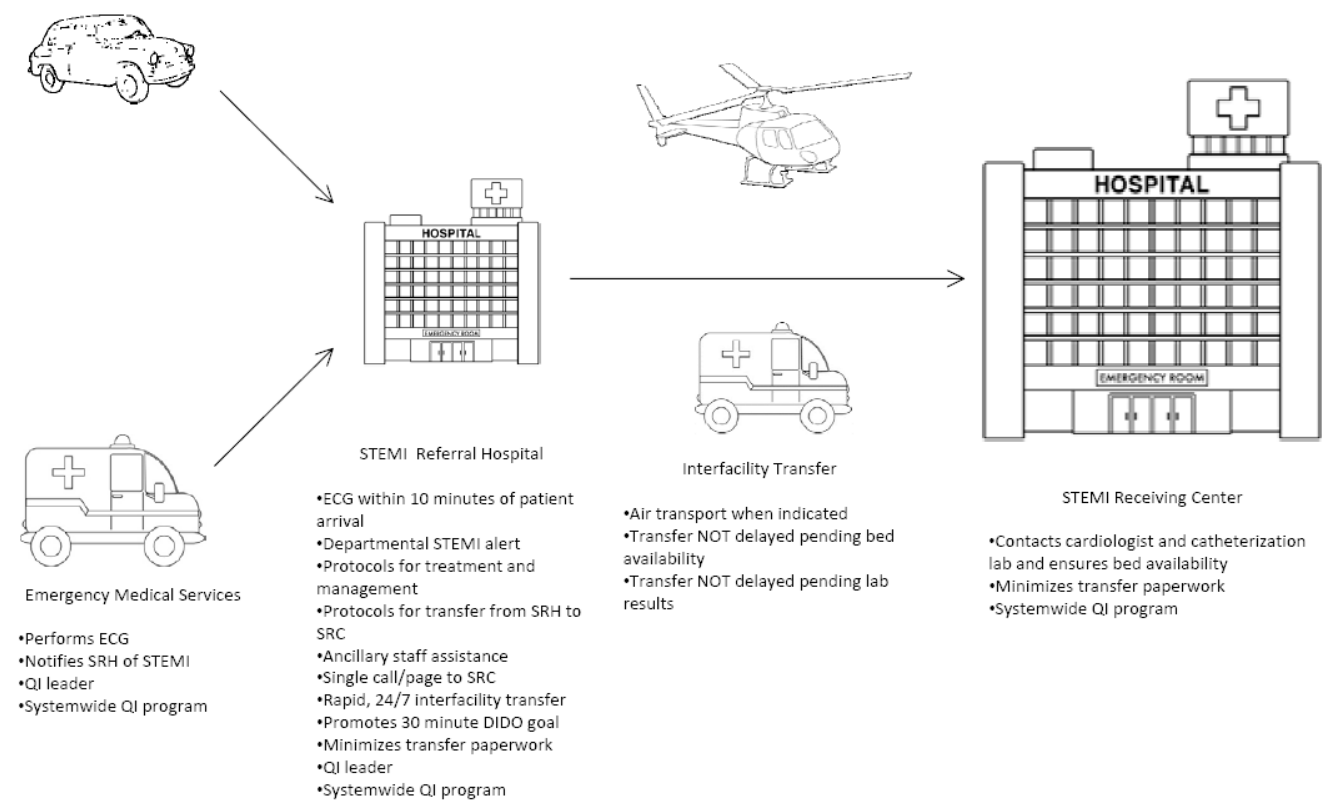

Figure 1.

Patient flow chart indicating the 18 consensus system practices.

Legend:

$\mathrm{ECG}=$ Electrocardiogram; STEMI = ST segment elevation myocardial infarction; SRH = STEMI referral hospital; SRC = STEMI receiving center; QI = Quality improvement; DIDO $=$ Door in door out 


\section{Table 1}

Participants by specialty in each round.

\begin{tabular}{|lccc|}
\hline Specialty & Round 1 & Round 2 & Round 3 \\
\hline EMS & 11 & 9 & 4 \\
Emergency Medicine & 10 & 7 & 6 \\
Interventional Cardiology & 7 & 5 & 3 \\
Other & 1 & 1 & 1 \\
\hline Total & 29 & 22 & 14 \\
\hline
\end{tabular}




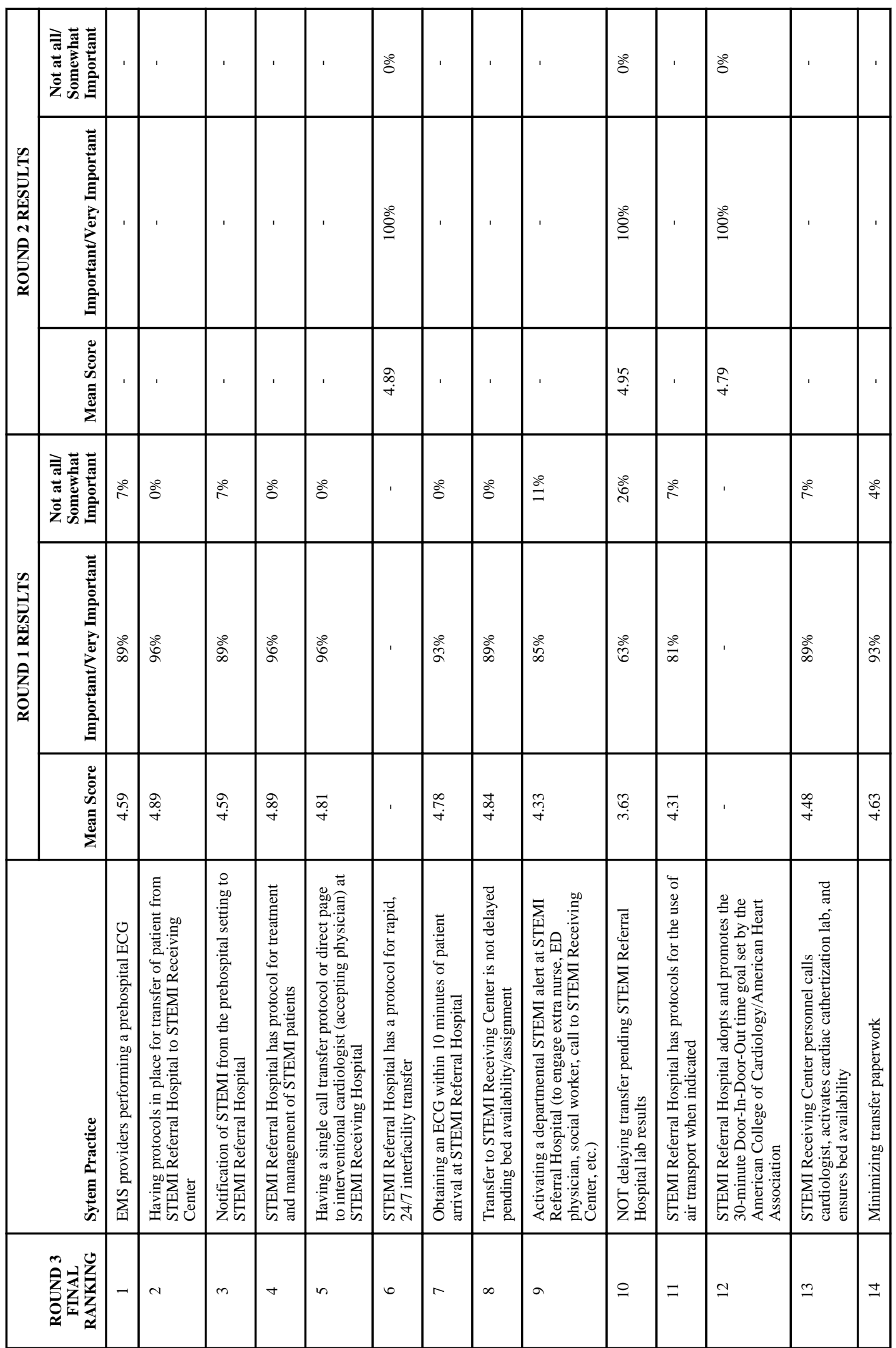




\begin{tabular}{|c|c|c|c|c|c|c|c|c|c|c|c|c|c|c|c|c|c|c|c|}
\hline & 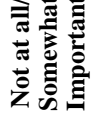 & i̊ & & $\therefore$ & 80 & & $\because 8$ & $\stackrel{\sim}{\circ}^{\circ}$ & : & $\stackrel{\circ}{\stackrel{2}{=}}$ & $\frac{\circ}{2}$ & & $\stackrel{\circ}{=}$ & $\stackrel{\circ 0}{=}$ & $\stackrel{\Xi}{=}$ & $\begin{array}{l}\infty \\
\infty \\
i\end{array}$ & $\ddot{i n}$ & $\begin{array}{c}0 \\
6\end{array}$ & $\stackrel{\diamond}{\sigma}$ \\
\hline 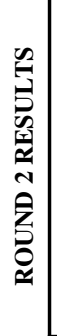 & 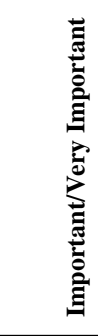 & $\check{\infty}_{\infty}^{\circ}$ & & $\stackrel{\circ}{\infty}$ & $\begin{array}{l}\stackrel{\circ}{\alpha} \\
\alpha\end{array}$ & & $\stackrel{\circ}{\infty}$ & $\infty_{0}^{\circ}$ & $\begin{array}{l}0 \\
\vdots \\
\infty \\
\infty\end{array}$ & Б̊ & $\begin{array}{l}\stackrel{0}{0} \\
0\end{array}$ & & $\begin{array}{l}\circ \\
0\end{array}$ & 过 & $\stackrel{\circ}{\circ}$ & $\stackrel{\circ}{0}$ & $\frac{\stackrel{\circ}{\infty}}{\infty}$ & $\frac{\stackrel{0}{\sim}}{\mathrm{v}}$ & $\stackrel{\circ}{=}$ \\
\hline & 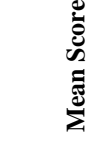 & $\begin{array}{l}\text { ণ } \\
\text { f }\end{array}$ & & 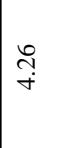 & $\begin{array}{l}\hat{\beta} \\
+ \\
+\end{array}$ & & $\underset{F}{\exists}$ & \begin{tabular}{|l}
+ \\
$\infty$ \\
$\dot{\sim}$
\end{tabular} & $\stackrel{\mathscr{\bullet}}{+}$ & $\overrightarrow{\mathcal{Y}}$ & $\begin{array}{l}\infty \\
\infty \\
\infty\end{array}$ & & $\stackrel{\vec{r}}{\dot{r}}$ & \begin{tabular}{|l}
$\infty$ \\
$\infty$ \\
$\dot{c o s}$
\end{tabular} & $\begin{array}{l}\hat{\sigma} \\
\dot{\sim}\end{array}$ & $\overrightarrow{\vec{i}}$ & $\stackrel{\stackrel{\leftrightarrow}{+}}{\dot{f}}$ & 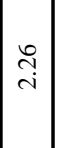 & ĩ \\
\hline & 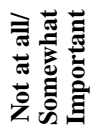 & & $\stackrel{\circ}{2}$ & & ' & & & $\frac{\stackrel{0}{m}}{m}$ & ते & ' & $\stackrel{\circ}{8}$ & & $\frac{\circ 0}{7}$ & $\stackrel{2}{2}$ & ठ্ল & $\stackrel{\stackrel{\circ}{ }}{\stackrel{\circ}{r}}$ & $\ddot{2}$ & & aे \\
\hline 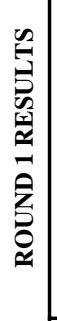 & 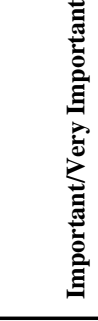 & & $\stackrel{\circ}{\circ}$ & & & & & $\begin{array}{l}0^{\circ} \\
\text { n }\end{array}$ & $\stackrel{\circ}{2}$ & ' & $\begin{array}{l}\stackrel{0}{\circ} \\
\text { ஸे }\end{array}$ & & $\begin{array}{l}\stackrel{0}{\sim} \\
i n\end{array}$ & 竎 & in & $\stackrel{\circ}{\circ}$ & $\stackrel{\stackrel{\circ}{+}}{ }$ & & ह్ర \\
\hline & 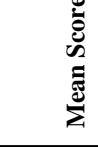 & & $\stackrel{m}{\rightarrow}$ & & & & & $\mid \begin{array}{l}\infty \\
\stackrel{\infty}{\infty} \\
\dot{m}\end{array}$ & $\mid \begin{array}{c}\infty \\
\infty \\
\dot{n}\end{array}$ & , & $\stackrel{\vartheta}{m}$ & & $\underset{\dot{m}}{\stackrel{a}{r}}$ & \begin{tabular}{|l}
$\infty$ \\
$\infty$ \\
$\infty$ \\
$\dot{c o s}$
\end{tabular} & $\underset{\sim}{\stackrel{J}{*}}$ & $\stackrel{\tilde{\Omega}}{-}$ & $\underset{\dot{F}}{\exists}$ & & के \\
\hline & 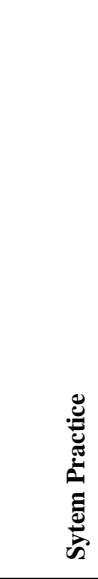 & 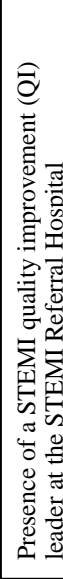 & 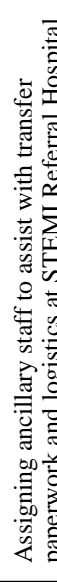 & 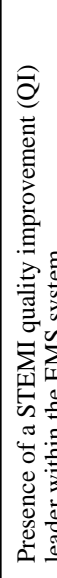 & 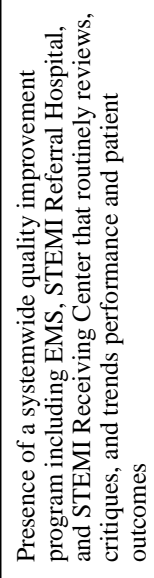 & 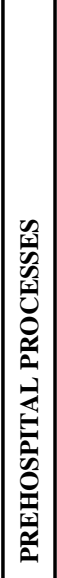 & 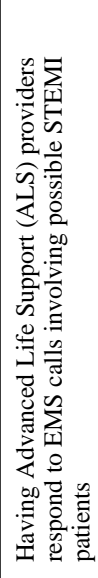 & 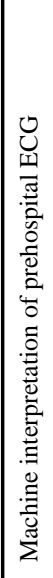 & 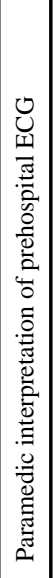 & 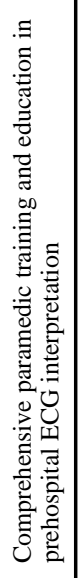 & 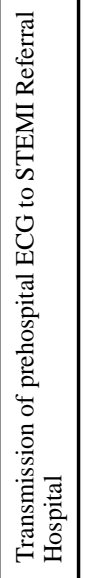 & 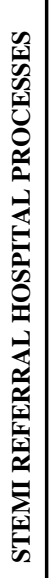 & 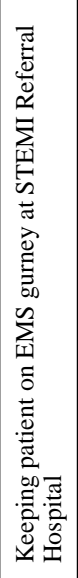 & 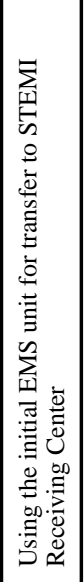 & 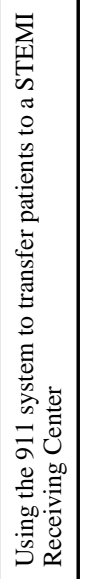 & 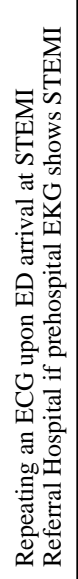 & 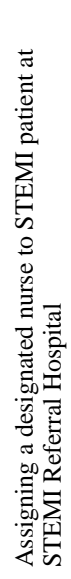 & 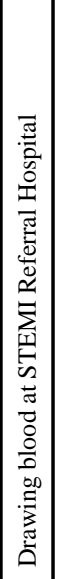 & 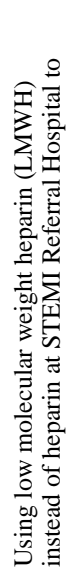 \\
\hline & 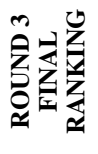 & $\simeq$ & $=1$ & $=$ & $\stackrel{\infty}{\infty}$ & & & & & & ' & & ' & ' & ' & & & & \\
\hline
\end{tabular}




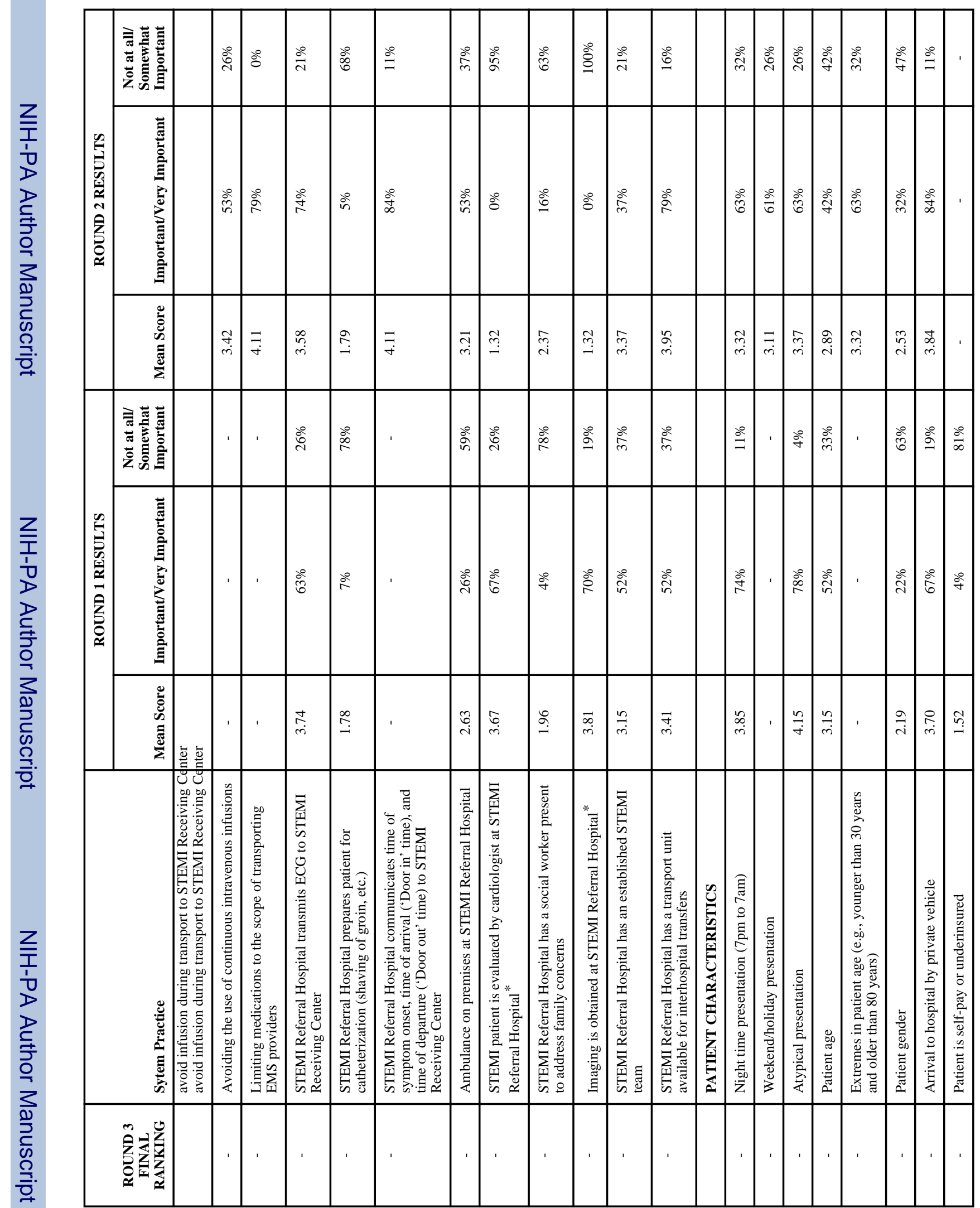




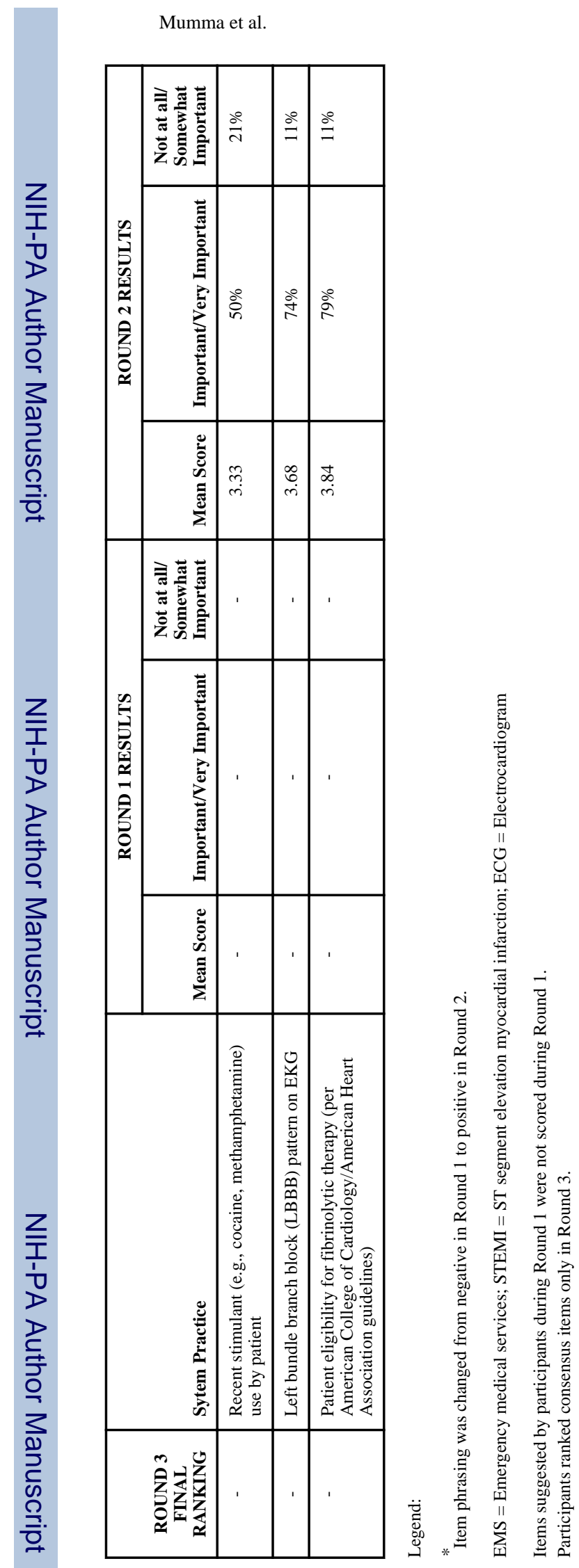

Page 12 